\title{
Al-doped $\mathrm{Nb}_{3} \mathrm{O}_{7} \mathrm{~F}$ nanosheets: preparation, characterization and photocatalytic performance under visible light irradiation
}

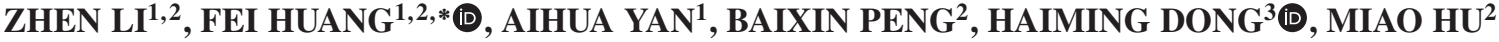 \\ and QI LI ${ }^{2}$ \\ ${ }^{1}$ Jiangsu Key Laboratory of Coal-based Greenhouse Gas Control and Utilization, Low Carbon Energy Institute, \\ China University of Mining and Technology, Xuzhou 221008, People's Republic of China \\ ${ }^{2}$ School of Materials Science and Engineering, China University of Mining and Technology, Xuzhou 221116, \\ People's Republic of China \\ ${ }^{3}$ School of Physics, China University of Mining and Technology, Xuzhou 221116, People's Republic of China \\ *Author for correspondence (huangfei7804@163.com)
}

MS received 15 May 2018; accepted 14 December 2018; published online 13 May 2019

\begin{abstract}
Doping can result in energy band rearrangement, high carrier concentration and fast carrier separation in semiconductor materials. Consequently, great enhancement in the photocatalytic performance can be achieved. In this work, $\mathrm{Al}^{3+}$-doped niobium oxyfluoride $\left(\mathrm{Nb}_{3} \mathrm{O}_{7} \mathrm{~F}\right)$ was synthesized by a facile hydrothermal approach for the first time. A series of characterization tools were used to investigate the phase, composition, microstructure, chemical state, spectrum response and band gap. The influence of Al doping on the photocatalytic performance was also investigated by varying the molar ratios of $\mathrm{Al} / \mathrm{Nb}$. The results confirmed that $\mathrm{Al}$ atoms have been successfully incorporated into the $\mathrm{Nb}_{3} \mathrm{O}_{7} \mathrm{~F}$ lattice. $\mathrm{Al}$ doping led to slightly lowering of the band gap of $\mathrm{Nb}_{3} \mathrm{O}_{7} \mathrm{~F}$ from 3.08 to $2.99 \mathrm{eV}$. Moreover, $\mathrm{Nb}_{3} \mathrm{O}_{7} \mathrm{~F}$ with $0.06 \mathrm{~mol} \% \mathrm{Al}$ doping exhibited the highest degradation efficiency for methyl blue under visible light irradiation. Crystal defects generated by appropriate Al-doping increased the specific surface area and the optimized band gap could be responsible for the improved photocatalytic activity.
\end{abstract}

Keywords. $\quad \mathrm{Nb}_{3} \mathrm{O}_{7} \mathrm{~F}$; Al-doping; band gap; photocatalysis.

\section{Introduction}

Photocatalysis is regarded as an effective technology for environmental control, and the key task is the preparation of highly efficient photocatalysts [1,2]. Among all semiconductor photocatalysts, $\mathrm{TiO}_{2}$ is the most popular due to its high $\mathrm{UV}$ photocatalytic efficiency and chemical inertness [3]. However, two typical drawbacks for $\mathrm{TiO}_{2}$ photocatalysts are low visible-light absorption and high carrier recombination [4]. In recent five years, $\mathrm{Nb}_{3} \mathrm{O}_{7} \mathrm{~F}$ (denoted as $\mathrm{NOF}$ ) nanomaterials with excellent photocatalytic properties, high melting point, stable phase, environmental benignity and low carrier recombination have been attracted considerable attention in degrading organic pollutants [5-7]. However, their wide bandgap $\left(E_{\mathrm{g}}=2.9 \sim 3.12 \mathrm{eV}\right)$ and low quantum efficiency result in poor photocatalytic activity and low overall efficiency $[8,9]$.

Doped modification, as an effective way to control band structure, improve spectrum adsorption, optimize electronic properties and enhance quantum efficiency, is promising in the development of efficient photocatalytic materials [10-12]. In particular, doping of various metal ions in photocatalysts, can not only facilitate the separation of photogenerated electronhole pairs and extend lifetimes of carriers, but also increase the spectrum response [13-15]. However, to date, there have been no reports on doped NOF. Considering that both $\mathrm{Al}-\mathrm{O}$ and $\mathrm{Nb}-\mathrm{O}$ have the same octahedral coordination structures and the ionic radius of $\mathrm{Al}(0.54 \AA)$ is smaller than that of $\mathrm{Nb}$ $(0.64 \AA)$, and the substitution of $\mathrm{Al}^{3+}$ for $\mathrm{Nb}^{5+}$ would be theoretically easy to realize $[16,17]$. Therefore, this allows us to achieve appropriate $\mathrm{Al}^{3+}$ content in doped samples according to the optimal photocatalytic performance.

In this work, we illustrate, for the first time, a facile hydrothermal method to synthesize Al-doped NOF nanomaterials using niobium pentachloride $\left(\mathrm{NbCl}_{5}\right)$ and aluminium trichloride $\left(\mathrm{AlCl}_{3}\right)$ as a precursor. The crystal phase and microstructure can be tuned by altering the $\mathrm{Al}^{3+}$ contents and determined by X-ray diffraction (XRD), field emission scanning electron microscopy (FESEM), high resolution transmission electron microscopy (HRTEM), energy dispersive spectrometer (EDS), X-ray photoelectron spectroscopy (XPS) and Raman spectroscopy. The influence of Al contents on photocatalytic activity has been investigated in detail. Furthermore, a potential model is proposed to explain the photocatalytic mechanism of Al-doped NOF.

\section{Experimental}

\subsection{Sample preparation}

All chemicals were analytical grade and used without further purification. Al-doped NOF nanosheets were synthesized 
using the following method as described in previous procedures $[6,18]$. In a typical procedure, $0.006 \mathrm{~mol} \mathrm{NbCl}_{5}$ (99.95\%, Alfa Aesar) and a certain amount of $\mathrm{AlCl}_{3}(99 \%$, Sinopharm Chemical Reagent Co. Ltd.) were dissolved in $80 \mathrm{ml}$ DI water, respectively. Afterwards, $0.0257 \mathrm{~mol}$ of hydrofluoric acid solution $(40 \%$, Sinopharm Chemical Reagent Co. Ltd.) was added to the above solution and stirred for several minutes. Then, the solution was transferred into a $100 \mathrm{ml}$ teflon-lined stainless autoclave, sealed, maintained at $180^{\circ} \mathrm{C}$ for $12 \mathrm{~h}$, and followed by natural cooling to room temperature. Finally, the precipitates were collected by centrifugation, washed several times and dried at $70^{\circ} \mathrm{C}$ for $5 \mathrm{~h}$. It should be stressed that Al-doped NOF nanosheets with different $\mathrm{Al} / \mathrm{Nb}$ molar ratios $(0.02,0.04,0.06$ and $0.08 \%)$ were designed and the as-synthesized samples were denoted as NOF-0.02A, NOF-0.04A, NOF-0.06A and NOF-0.08A, respectively.

\subsection{Characterization}

XRD (Bruker D8) with $\mathrm{CuK} \alpha$ radiation was used to analyse the crystal phase of the as-synthesized Al-doped NOF. The scan rate was $5^{\circ} \mathrm{min}^{-1}$ in the $2 \theta$ range of $10-90^{\circ}$. The morphology and size were observed by FESEM (Hitachi S4800). HRTEM (FEI, Tecnai G2 F20) was used to investigate the micro-morphology and crystal lattice of final samples. Element distribution was analysed by EDS (Thermo Noran VANTAG-ESI). XPS (Escalab 250Xi) was applied to characterize the chemical state of the final samples. The information of the molecular structure was recorded with Raman spectroscopy with an excitation wavelength of $532 \mathrm{~nm}$. The absorption spectra were recorded by a UV-Vis spectrometer (Analytik Jena specord®210) with an integrating sphere of $75 \mathrm{~mm}$.

\subsection{Photocatalytic procedure}

The photocatalytic activity of the as-prepared Al-doped NOF was evaluated by performing the experiments in aqueous methyl blue (MB) solution under visible light irradiation, and emitted by a Xe lamp with two cutoff filters (JB380 and VISREF350-780). Typically, $20 \mathrm{mg}$ Al-doped NOF photocatalysts were added to $200 \mathrm{ml} \mathrm{MB}$ solution $\left(10 \mathrm{mgl}^{-1}\right)$ and transferred to a designed reaction vessel. The chamber was constantly stirred for $1 \mathrm{~h}$ in the dark to achieve adsorptiondesorption equilibrium. During irradiation, $5 \mathrm{ml}$ suspension was periodically taken out, centrifuged to remove the photocatalyst, and analysed through monitoring the change of absorbance at $665 \mathrm{~nm}$. The degradation efficiency (\%) was calculated as follows:

$$
\text { Efficiency }(\%)=\left(C_{0}-C_{\mathrm{t}}\right) / C_{0} \times 100,
$$

where $C_{0}$ and $C_{\mathrm{t}}$ are the concentrations of MB solution before and after irradiation, respectively.

\section{Results and discussion}

The XRD patterns of pure NOF and NOF-0.06A samples were recorded to investigate the changes of the crystal structure. As shown in figure 1a, all the peaks for pure NOF and NOF-0.06A samples are identical to orthorhombic NOF (JCPDS no. 74-2363) without any impurity phases. The main eight peaks at 22.7, 23.6, 25.9, 26.8, 31.9, 33.1, 46.2 and $47.5^{\circ}$ can be attributed to the (001), (110), (600), (310), (510), (111), (002) and (020) crystalline planes of orthorhombic NOF. The inset shows that the (001) peak of Al-doped NOF shifts to a slightly higher angle than that of pure NOF. The results may be caused by slightly structural deformation when $\mathrm{Al}$ atoms are incorporated into the NOF crystal lattice [19].

Raman spectroscopy is a kind of effective tool to characterize the molecular structure. As shown in figure $1 \mathrm{~b}$, pure NOF and NOF-0.06A samples have four characteristic modes, which follow the $\mathrm{ReO}_{3}$ type symmetry [20]. The peaks at $73.7,222.2$ and $707.9 \mathrm{~cm}^{-1}$ correspond to $\mathrm{O}=\mathrm{Nb}-\mathrm{O}-\mathrm{F}$ vibration mode, $\mathrm{O}=\mathrm{Nb}=\mathrm{O}$ twisting mode and $\mathrm{Nb}-\mathrm{O}-\mathrm{Nb}$ stretching vibration mode, respectively $[18,21]$. However, the peak at $126.5 \mathrm{~cm}^{-1}$ ascribed to $\mathrm{Nb}-\mathrm{F}$ vibration mode tends to be
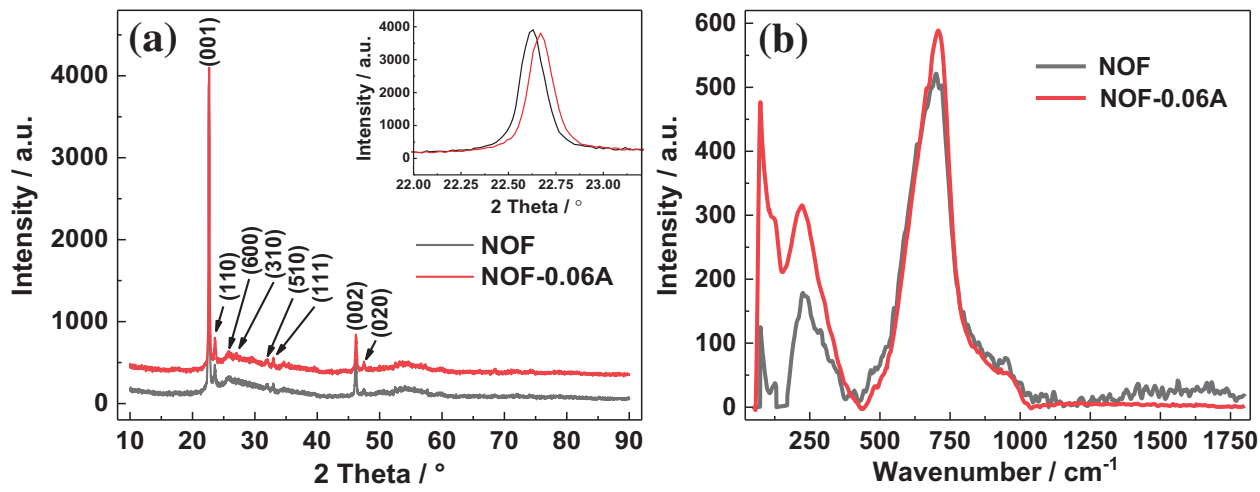

Figure 1. (a) XRD patterns and (b) Raman spectra of the synthesized samples. 
broadened for Al-doped NOF samples [22]. The change may result from the structural disorder induced by the substitution of $\mathrm{Al}$ for $\mathrm{Nb}$, which further confirms the XRD result. The EDS result of pure NOF shows that the samples only contain $\mathrm{O}, \mathrm{F}$ and $\mathrm{Nb}$ elements (figure $2 \mathrm{a}$ ), while NOF-0.06A samples have Al element except above three elements (figure $2 \mathrm{~b}$ ). The results further imply the possible doping of $\mathrm{Al}$ element in NOF.
The morphological changes between pure NOF and Al-doped NOF samples were investigated by FESEM and HRTEM. It is clearly found that Al-doping plays an important role in the morphological evolution. As shown in figure 3a, pure NOF samples are dominated by microspheres with a diameter of $8-10 \mu \mathrm{m}$. The magnified image shows that the microspheres are composed of many nanosheets with a thickness of about $50 \mathrm{~nm}$ and a width of $1-2 \mu \mathrm{m}$ (figure $3 \mathrm{~b}$ ).

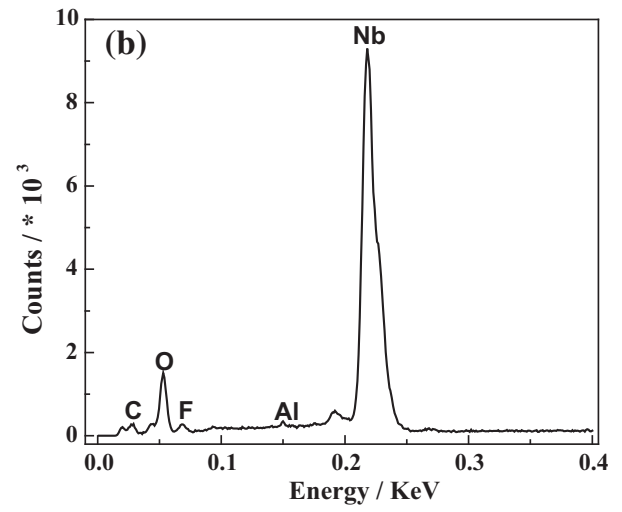

Figure 2. EDS patterns of (a) pure NOF and (b) NOF-0.06A.
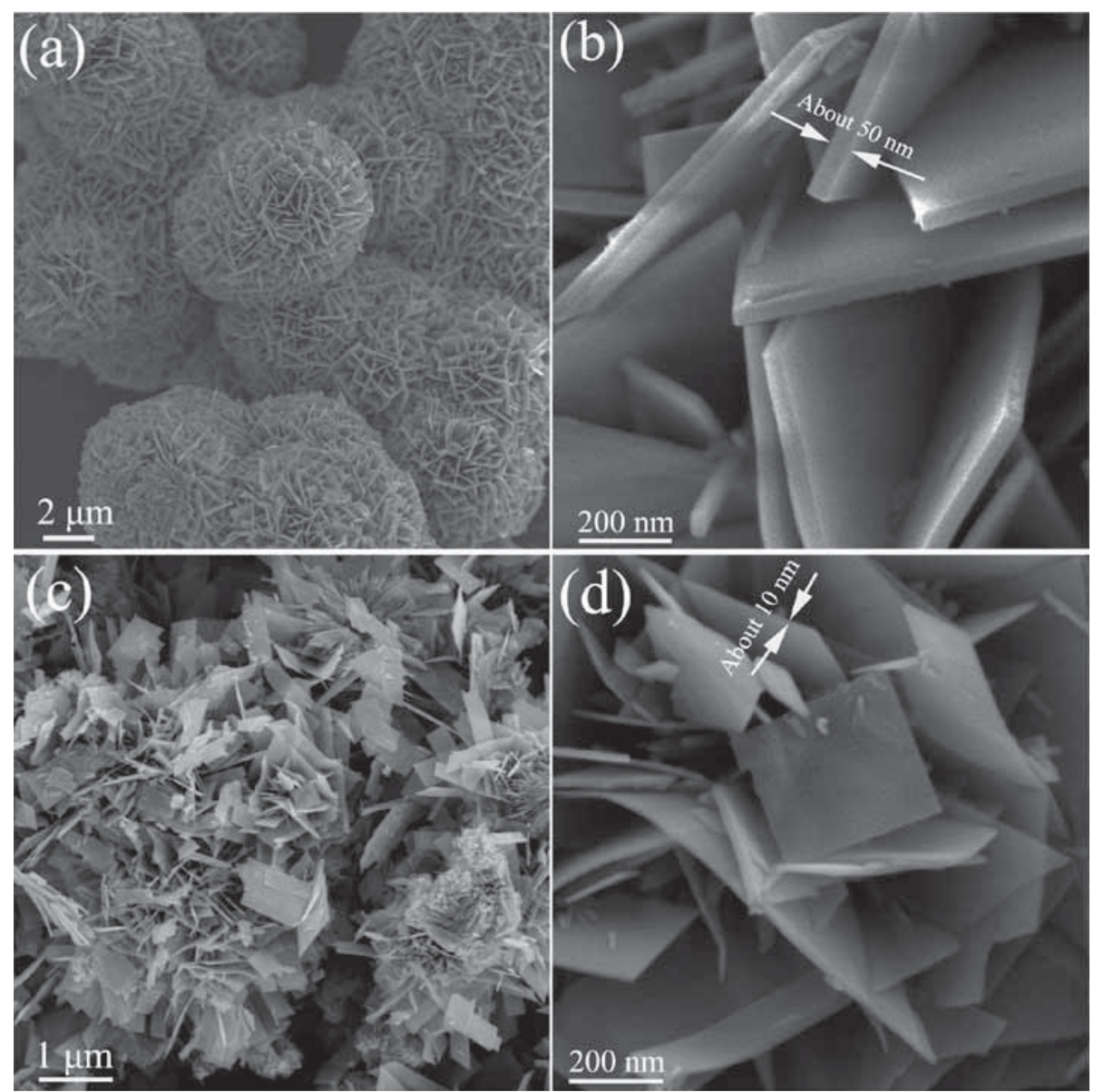

Figure 3. FESEM images of $(\mathbf{a}, \mathbf{b})$ pure NOF and $(\mathbf{c}, \mathbf{d})$ NOF-0.06A. 
After Al-doping, the microspheres gradually disappear and continuously evolve to the aggregates of nanosheets with a thickness of about $10 \mathrm{~nm}$ (figure $3 \mathrm{c}$ and d). In other words, the distribution of Al-doped NOF becomes more irregular and the thickness of the nanosheet becomes thinner. The HRTEM image shows that the final samples of NOF-0.06A are composed of many nanosheets, which are in agreement with the FESEM results (figure 4a). The HRTEM image shows that the nanosheets have good crystallinity (figure $4 b$ ). The distance between lattice planes along the nanosheet growth is $0.396 \mathrm{~nm}$, which corresponds to the (001) $d$-spacing of NOF. The results indicate that the nanosheet preferentially grows along the [001] direction, which is in well agreement with pure NOF and the previous report [18]. On the other
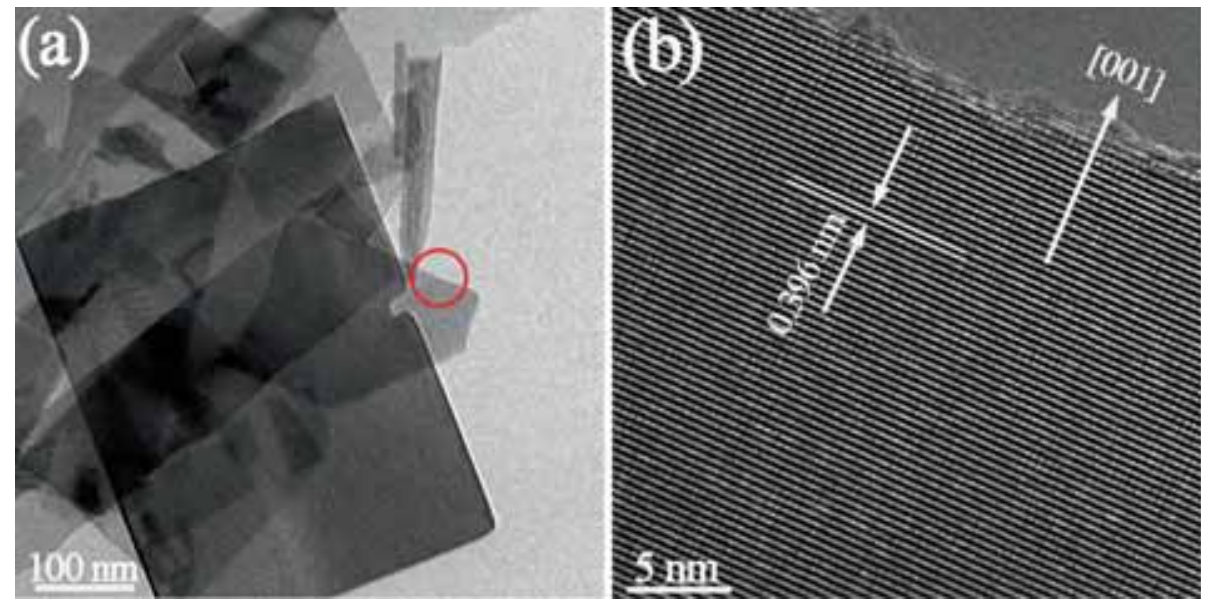

Figure 4. TEM images of the NOF-0.06A sample. (a) Low-magnified image and (b) highresolution image.
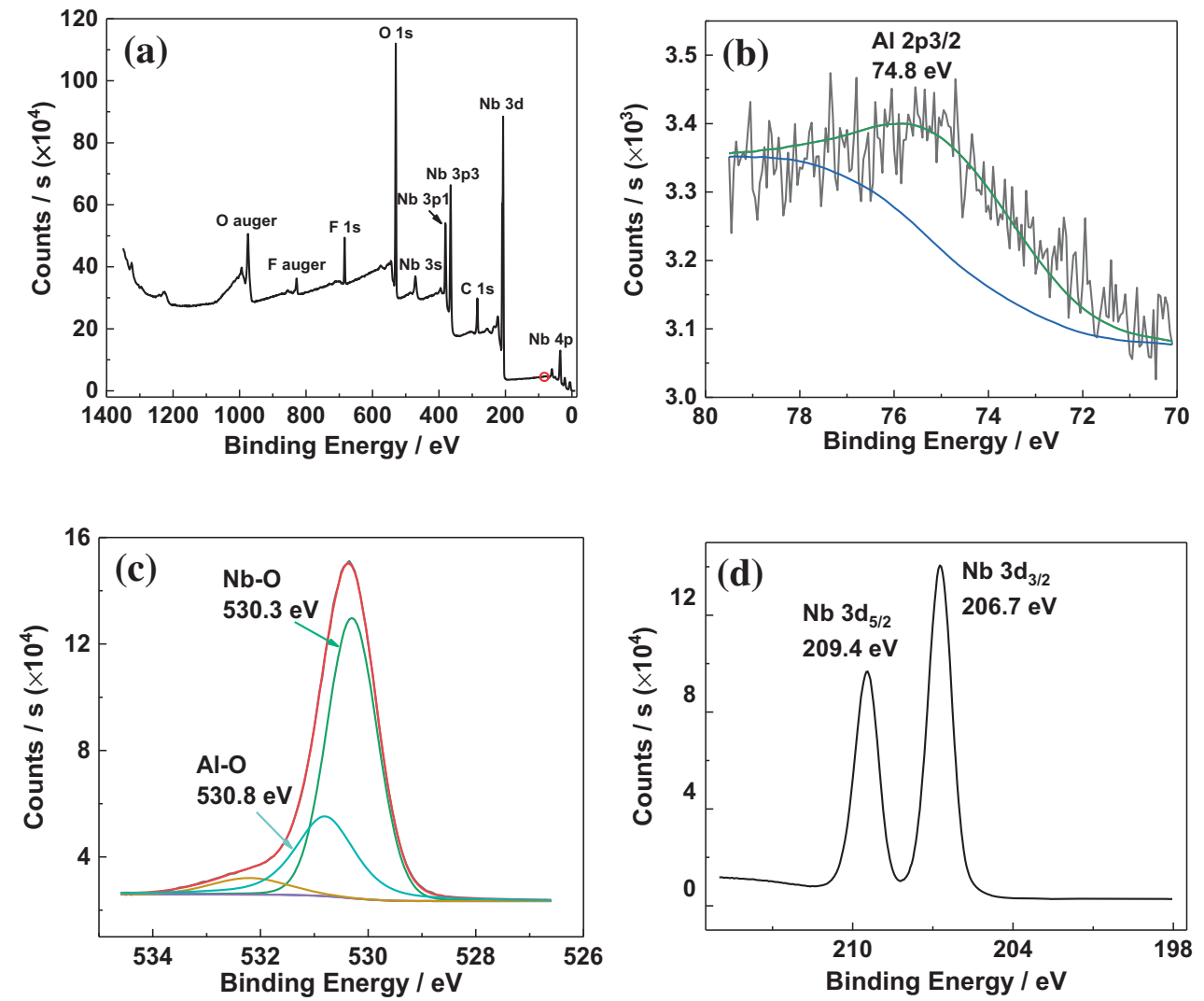

Figure 5. XPS spectra of NOF-0.06A samples. (a) Wide scan; (b) narrow-scanned Al 2p; (c) narrowscanned $\mathrm{O} 1 \mathrm{~s}$ and (d) narrow-scanned $\mathrm{Nb} 3 \mathrm{~d}$. 
hand, the results also indicate that Al-doping has no obvious influence on the phase transition due to low $\mathrm{Al}$ content.

As shown in figure $5 \mathrm{a}, \mathrm{Nb}, \mathrm{F}$ and $\mathrm{O}$ elements in the widescan XPS spectrum can clearly be observed for NOF-0.06A samples. We also studied narrow-scanned XPS spectra for chemical analysis of $\mathrm{Nb}, \mathrm{O}$ and $\mathrm{Al}$ elements. As shown in figure $5 \mathrm{~b}$, the peak at $74.8 \mathrm{eV}$ can be assigned to $\mathrm{Al} 2 \mathrm{p}_{3 / 2}$, which indicates the existence of $\mathrm{Al}^{3+}$ ions. The narrowscanned spectrum of $\mathrm{O} 1 \mathrm{~s}$ can be fitted into the two obvious peaks at 530.8 and $530.3 \mathrm{eV}$, which are attributed to $\mathrm{Al}-\mathrm{O}$ and $\mathrm{Nb}-\mathrm{O}$, respectively (figure $5 \mathrm{c}$ ) [23]. The result indicates that $\mathrm{Al}$ atoms have been successfully incorporated into the NOF lattice. The peaks at 209.4 and $206.7 \mathrm{eV}$ match the binding energy of $\mathrm{Nb}_{3 / 2} \mathrm{~d}_{5 / 2}$ and $\mathrm{Nb}_{3} \mathrm{~d}_{3 / 2}$ (figure $5 \mathrm{~d}$ ), indicating the $\mathrm{Nb}^{5+}$ oxidation state [24].

The influence of Al-doping on the spectrum response and band gap was analysed and calculated from UV-Vis spectra. As shown in figure $6 a$, the optical absorption edges for
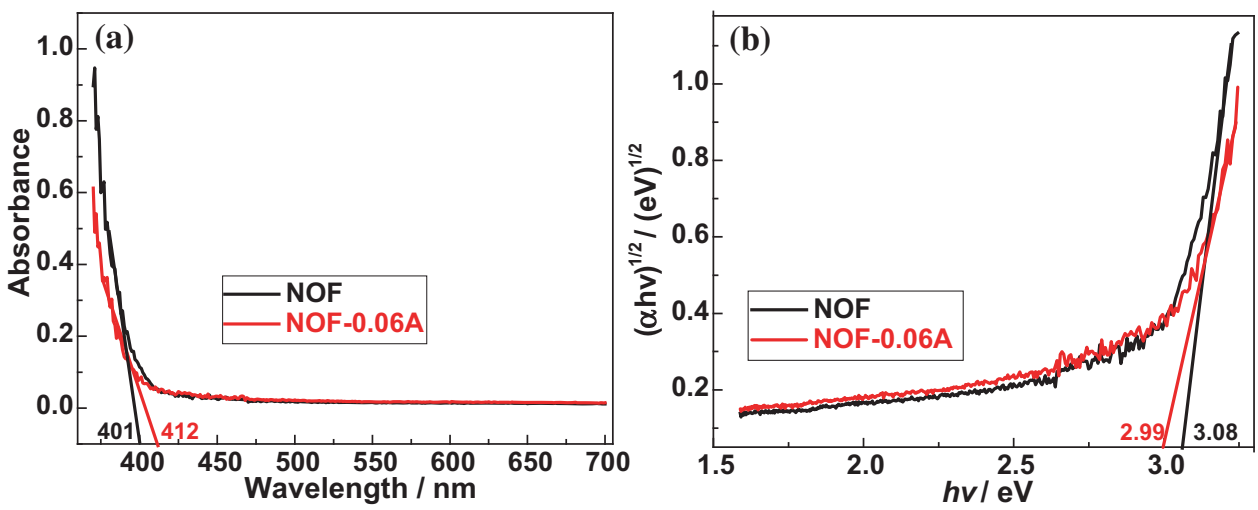

Figure 6. (a) UV-Vis absorption spectra and (b) $(\alpha h v)^{1 / 2} v s$. $h v$ curves of pure NOF and NOF-0.06A samples.
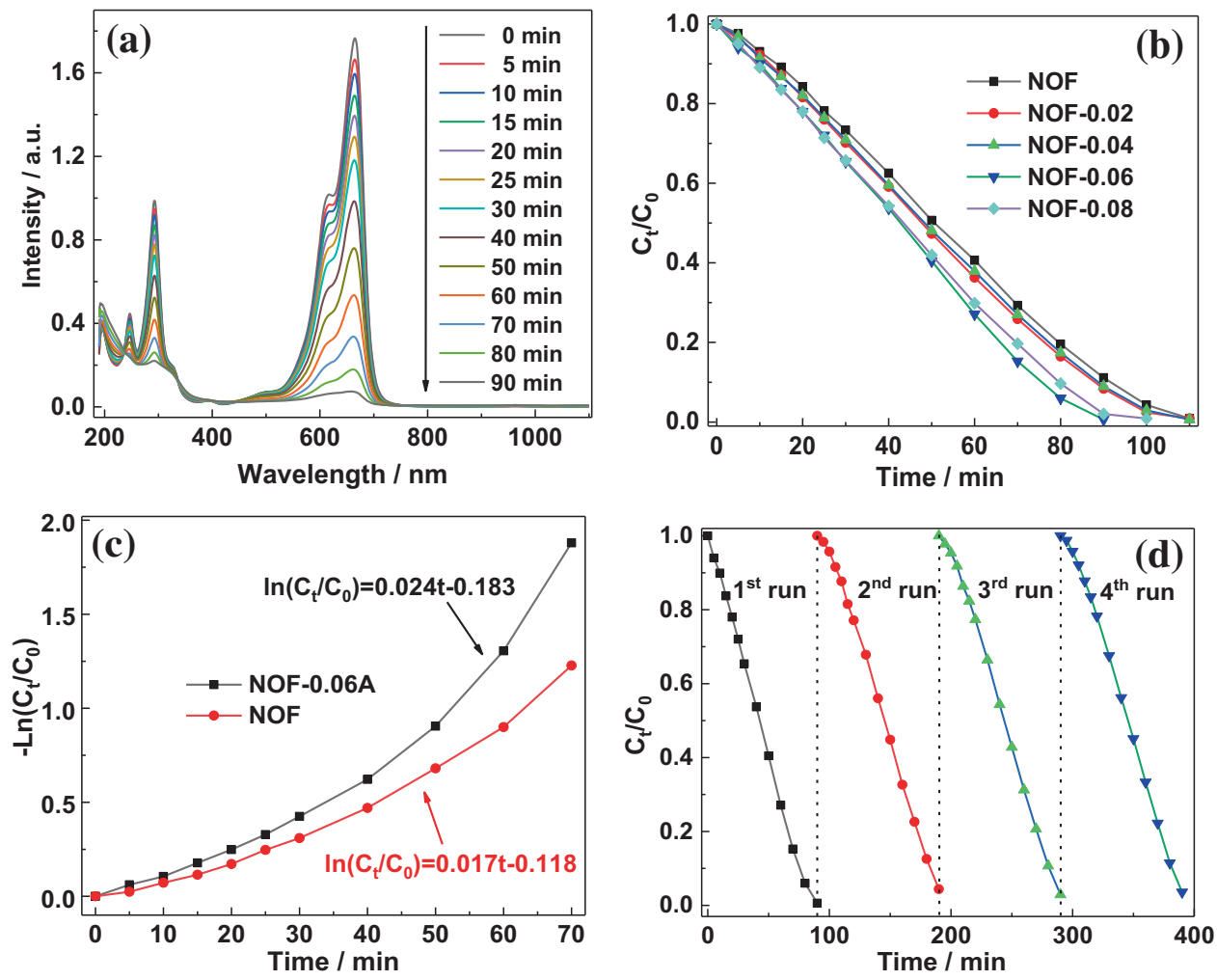

Figure 7. Photocatalytic degradation curves of different samples. (a) Temporal UV-Vis spectra in the presence of NOF-0.06A photocatalysts; (b) photocatalytic degradation vs. time curves; (c) photocatalytic rate curves and (d) repeated curves of photocatalytic degradation. 
pure NOF and NOF-0.06A limit to about 401 and $412 \mathrm{~nm}$, respectively. According to the Kubelka-Munk relation [25], the calculated band gaps are 3.08 and $2.99 \mathrm{eV}$ (figure 6b), respectively. The result reveals that $\mathrm{Al}$ doping results in a slight reduction of the band gap. Such results can potentially deduce that $\mathrm{Al}^{3+}$ incorporated into the NOF matrix, which is in agreement with the results of XRD and Raman.

The photocatalytic performances of pure NOF and Aldoped NOF samples were evaluated through the degradation of MB solution under visible light irradiation (380 $\mathrm{nm} \leq \lambda \leq$ $780 \mathrm{~nm}$ ). As presented in figure 7a, MB can be degraded with extending the degradation time in the presence of NOF-0.06A photocatalysts and the characteristic peak at $665 \mathrm{~nm}$ decreases gradually. The best photocatalytic activity can be observed for NOF-0.06A samples (figure 7b). The degradation ratio of
MB is about $99.4 \%$ within 90 min, but only $88.9 \%$ removal for pure NOF samples. Moreover, the photodegradation of $\mathrm{MB}$ is found to follow the pseudo-first-order kinetic model:

$$
-\ln \left(C_{\mathrm{t}} / C_{0}\right)=k t
$$

where $k$ is the degradation rate constant and $t$ the irradiation time. As shown in figure $7 \mathrm{c}$, the rate constant of NOF-0.06A at $0.024 \mathrm{~min}^{-1}$ within $70 \mathrm{~min}$, which is higher than that of pure NOF $\left(k=0.017 \mathrm{~min}^{-1}\right)$. Furthermore, the NOF-0.06A sample still maintains good photocatalytic activity with no obvious decay after four cycling experiments (figure 7d). The results imply that the Al-doped NOF has good repeativity and stability.
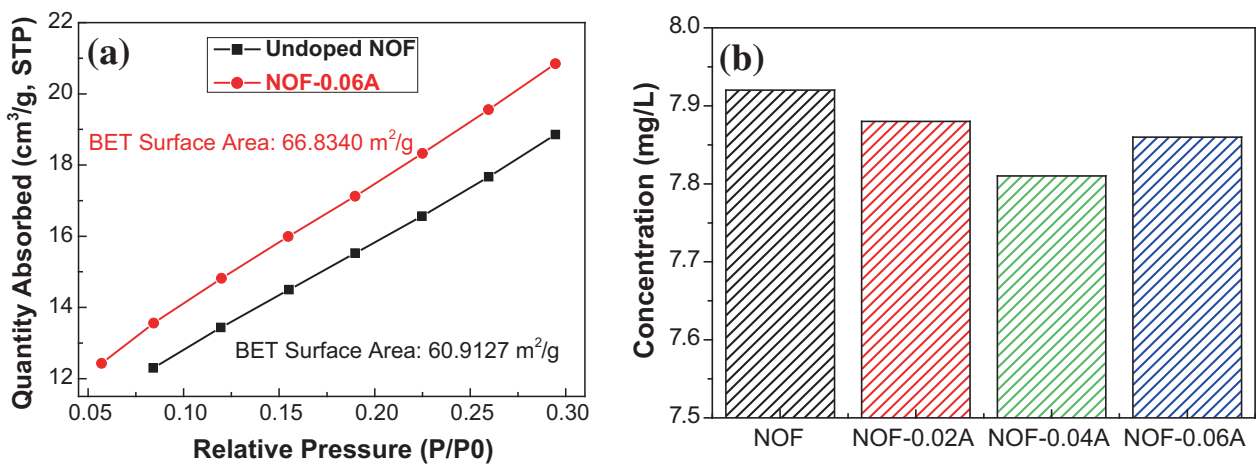

Figure 8. (a) Nitrogen adsorbed quantity vs. relative pressure curves and (b) adsorption-desorption balance concentrations of MB for different samples.

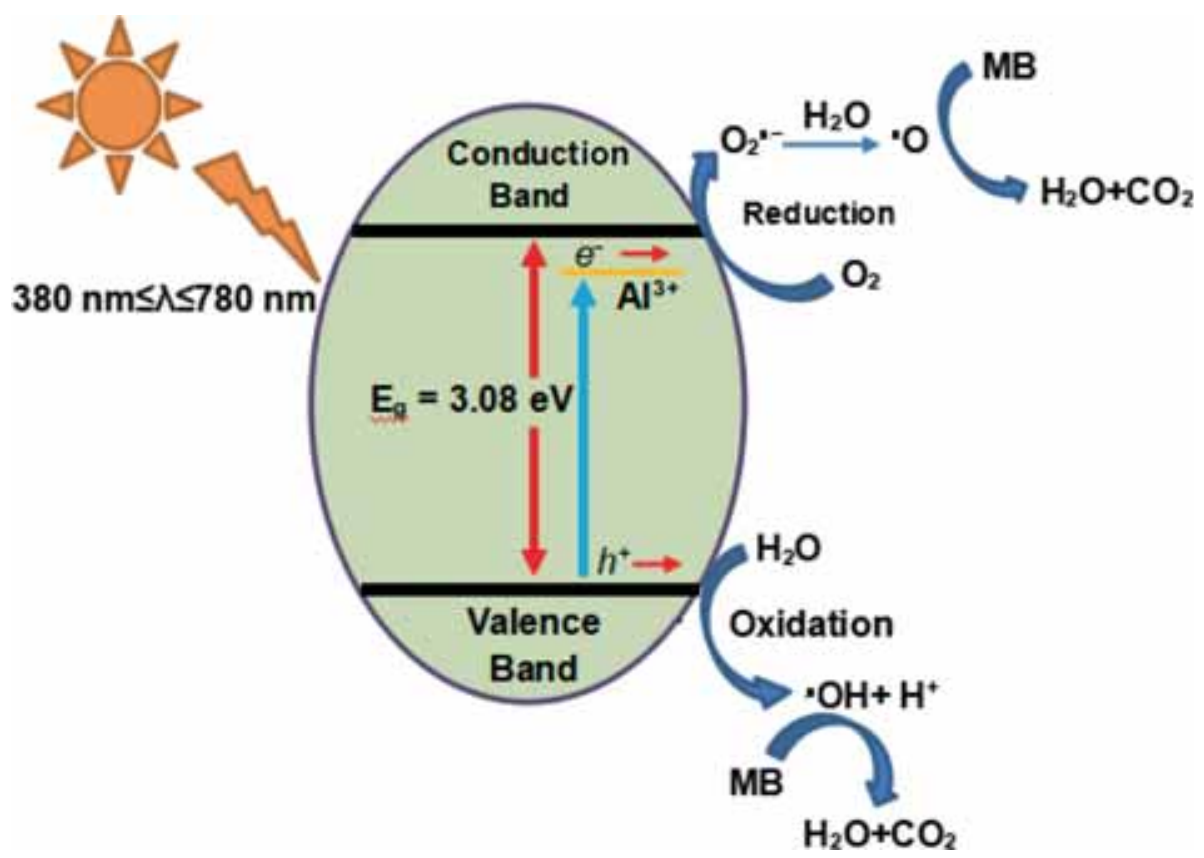

Figure 9. Schematic diagram of the photocatalytic mechanism for Al-doped NOF. 
Based on the above experimental results, a potential photocatalytic mechanism is proposed to explain the enhanced photocatalytic activity of Al-doped NOF. Firstly, Al-doped NOF nanosheets with a larger specific area can potentially enhance the adsorption capacity and provide more active sites. Nitrogen adsorption shown in figure $8 \mathrm{a}$ further confirms that Al-doped NOF photocatalysts with $66.934 \mathrm{~m}^{2} \mathrm{~g}^{-1}$ have a larger surface area than pure NOF with $60.9127 \mathrm{~m}^{2} \mathrm{~g}^{-1}$. As presented in figure $8 \mathrm{~b}$, it can also be clearly observed that Al-doped NOF nanosheets have lower adsorption-desorption balance concentration, further indicating that Al-doped NOF has higher adsorption ability. Secondly, the substitutional Al atoms result in the formation of defects, which enhances the carrier concentration and charge separation efficiency [26]. Moreover, Al impurity can act as transport channels to promote the transfer of photogenerated electrons and suppress the recombination of carriers. Thirdly, Al-doping can narrow the band gap of NOF and introduce isolated states below the conduction band (CB) edge. Consequently, electrons can be excited to the $\mathrm{CB}$ from the valence band under the visible light irradiation. That is, electron-hole pairs easily form after Al-doped NOF photocatalysts absorb sufficient energy. Finally, a majority of excited electrons resides on the states formed by $\mathrm{Al}^{3+}$ ions and reacts with surface $\mathrm{O}_{2}$ to produce superoxide anions. In the meantime, hydroxyl radicals with strong oxidizability also generate through the reaction of holes and surface water to oxidize organic pollutants. Finally, $\mathrm{MB}$ is oxidized and degraded gradually into small inorganic molecules, such as $\mathrm{H}_{2} \mathrm{O}$ and $\mathrm{CO}_{2}$ [27,28]. The schematic illustration of Al-doped NOF photocatalysts is presented in figure 9.

\section{Conclusions}

In summary, Al-doped NOF nanosheets have been successfully synthesized by a facile hydrothermal method. The average thickness of nanosheets slightly decreases from 50 to $10 \mathrm{~nm}$ after Al-doping. After $\mathrm{Al}$ atoms are incorporated into the NOF crystal, little structural deformation is observed. $\mathrm{UV}-\mathrm{Vis}$ shows that Al-doping results in slight reduction of the band gap from 3.08 to $2.99 \mathrm{eV}$. The NOF-0.06A samples exhibit the highest degradation efficiency of $99.6 \%$ under visible light irradiation within $90 \mathrm{~min}$, which is obviously higher than that of pure NOF. The enhanced photocatalytic activity can be attributed to the larger specific surface area, higher photogenerated electron-hole separation and lower carrier recombination. Furthermore, Al-doped NOF photocatalysts have good repeativity and stability within four cycling tests.

\section{Acknowledgements}

The work was financially supported by the Fundamental Research Funds for the Central Universities (2017XKQY010).
The Huang group acknowledges the support from the Advanced Analysis and Computation Center of CUMT.

\section{References}

[1] Tong H, Ouyang S, Bi Y, Umezawa N, Oshikiri M and Ye J 2012 Adv. Mater. 24229

[2] Wang H, Zhang L, Chen Z, Hu J, Li S, Wang Z et al 2014 Chem. Soc. Rev. 435234

[3] Ma Y, Wang X, Jia Y, Chen X, Han H and Li C 2014 Chem. Rev. 1149987

[4] Zhang L, He X, Xu X, Liu C, Duan Y, Hou L et al 2017 Appl. Catal. B Environ. 2031

[5] Idrees F, Chao C, Butt F K, Yahir M, Tanveer M, Aslam I et al 2013 CrystEngComm 158146

[6] Huang F, Li Z, Yan A, Zhao H, Feng H and Wang Y 2017 Nanotechnology 28275707

[7] Wang Z, Hou J, Yang C, Jiao S, Huang K and Zhu H 2013 Phys. Chem. Chem. Phys. 153249

[8] Duan J, Mou G, Zhang S, Wang S and Guan J 2015 J. Mater. Chem. A 314686

[9] Zhang H, Li Y, Wang Y, Liu P, Yang H, Yao X et al 2013 J. Mater. Chem. A 16563

[10] Li Z, Huang F, Feng X, Yan A, Dong H, Hu M et al 2018 Nanotechnology 29225605

[11] Liu M, Qiu X, Miyauchi M and Hashimoto K $2013 \mathrm{~J}$. Am. Chem. Soc. 13510064

[12] Phuruangrat A, Cheed-Im U, Thongtem T and Thongtem S 2016 Mater. Lett. 172166

[13] Wu Y, Huang Y and Yang H 2016 CrystEngComm 186881

[14] Gong G, Liu Y, Mao B, Tan L, Yang Y and Shi W 2017 Appl. Catal. B Environ. 21611

[15] Zhang M, Hu C, He X, Wan B and Xi Y 2009 Catal. Commun. 11206

[16] Iwakura H, Einaga H and Teraoka Y 2010 Inorg. Chem. 49 11362

[17] Andersson S 1964 Acta Chem. Scand. 182339

[18] Huang F, Li Z, Yan A, Zhao H, Liang H, Gao Q et al 2017 Sci. Rep. 739973

[19] Zhang W, Pei X, Chen J and He H 2015 Mater. Sci. Semicond. Process. 3824

[20] Huang F, Li Z, Yan A, Zhao H, Feng H, Hu M et al 2018 Adv. Mater. Interfaces 51701304

[21] Huang F, Zhao H, Feng X, Yan A, Chen G, Li Z et al 2017 Ceram. Int. 432743

[22] Chouchene B, Chaabane T B, Mozet K, Girot E, Corbel S, Balan L et al 2017 Appl. Surf. Sci. 409102

[23] Huang F, Yan A, Liao Z, Zhao H, Fu Z, Zhang F et al 2015 Mater. Technol. 30144

[24] Wu W, Liang S, Shen L, Ding Z, Zheng H, Su W et al 2012 J. Alloys Compd. $\mathbf{5 2 0} 213$

[25] Kubelka P and Munk F 1931 Z. Tech. Phys. 12593

[26] Bensouici F, Bououdina M, Dakhel AA, Souier T, Tala-Ighil R, Toubane M et al 2016 Thin Solid Films 616655

[27] Ammar H, Hinda L, Mohamed K, Elimame E, Chantal G and Jean-Marie H 2001 Appl. Catal. B Environ. 31145

[28] Dariani R S, Esmaeili A, Mortezaali A and Dehghanpour S 2016 Optik 1277143 